\title{
Integrated broadband antenna for USB digital Terrestrial TV receiver
}

\author{
Philippe Minard, Jean-François Pintos and Ali Louzir \\ Thomson Technology Group - Corporate Research \\ Network and Communication Laboratory, \\ Thomson R\&D France, 1 Avenue Belle Fontaine, Cesson Sévigné, 35576, France. \\ http://www.thomson.net
}

\section{Introduction}

With the recent arrival of digital terrestrial TV in several major countries, there is an increasing interest in developing a compact light weight USB-stick like device allowing the reception of free to air digital TV programming by connecting it directly to an USB port of a PC or a laptop. Basically, the receiver includes three Integrated Circuits (ICs): an RF Silicon tuner for channel selection, a digital IC for signal demodulation and an USB adaptor chip. The MPEG demodulated Transport Stream is then decoded by software previously loaded on your PC or laptop.

However most of the products are supplied with an external terrestrial whip type portable antenna that should be connected thru a coaxial cable to UHF/VHF port of the USB stick [1]. This limits the portability and the mobile use of such a product.

In this paper, it is proposed an USB stick with integrated antenna where the housing of the compact receiver is used as part of a wideband UHF antenna working over the full [470-862MHz] band as suggested by the European standard [2]. It results a small and light weight pocket-sized receiver very attractive for portable use.

\section{Antenna description}

Figure 1 shows a photograph and the dimensions of the USB stick receiver excluding the antenna, etched on a classical low-cost multi-layer FR4 substrate. The idea is to design a wideband dipole-type antenna with one arm used as housing of the USB stick receiver. The length of each arm is chosen approximately equal to the quarter wavelength at the central frequency of the targeted UHF-band.

A 3D perspective of the proposed antenna is presented in Figure 2. The volume of the first arm is sized so that it includes the USB stick receiver while it insures a good starting point for the antenna design optimization. The second arm with flat and curved sections is connected to the input of the receiver. It could be folded along the base of the PCB housing, when the USB stick is not in use. The curved section around the dipole feeding point is optimized so that to insure sufficient antenna matching over the full UHF-band. The total length of both arms is approximately equal to the half wavelength at the central frequency. 
Finally, the various positions of the second foldable arm (Figure 2) allow some optimizations of the frequency response and radiation pattern orientation of the antenna, depending on reception conditions and the selected UHF channel.

\section{Design optimization and obtained results}

The dimensions of the obtained asymmetric dipole antenna have been optimized by simulations using IE3D software. Figure 3 shows the final dimensions of the antenna and Figure 4 presents the simulated antenna return loss for various folding angle $\alpha$ of the second arm. It could be noticed that the resonant frequency of the antenna varies from $600 \mathrm{MHz}$ for $\alpha=0^{\circ}$, to $710 \mathrm{MHz}$ for $\alpha=90^{\circ}$ allowing some frequency adjustment flexibility. However, in order to achieve a good antenna efficiency (efficiency $>75 \%$ ), the antenna return loss should be better than $-6 \mathrm{~dB}$ over the full UHF bandwidth. For that purpose, a matching circuit is inserted between the antenna and the input of the LNA. Figure 6 shows the obtained antenna return losses for the various folding angles when one cell $\mathrm{LC}$ matching circuit (shown in Figure 5 with $\mathrm{C}=5 \mathrm{pF}$ and $\mathrm{L}=15 \mathrm{nH}$ ) is used. It could be noticed that the targeted return loss of $-6 \mathrm{~dB}$ is achieved over the whole UHF band for $\alpha<=60^{\circ}$. Figure 7 presents the simulated efficiency of the antenna with $\alpha$ as parameter.

Finally, the simulated radiation patterns of the antenna for various folding angle $\alpha$ are presented in Figure 8, confirming its dipole-type behavior.

The designed antenna has been realized using metallized plastic technology. Figure 9 shows a photograph of the antenna including a DVB-T integrated receiver. Figure 10 shows the measured return loss compared with simulated results, showing good agreement between simulations and measurements.

The realized USB stick receiver with built-in antenna has been successfully used for the terrestrial DVB-T reception in Europe.

\section{Conclusion}

A built-in antenna for an USB key like digital Terrestrial TV receiver allowing free to air reception of digital Terrestrial TV on a computer PC have been designed, realized and tested. The housing of the compact receiver is used as part of the antenna. The antenna design is optimized so that it covers the whole UHF frequency bandwidth with good radiation efficiency, while its size is imposed by the USB-key form factor. The obtained results are compliant with simulations.

\section{References}

\section{[1] http://images.google.com/images?q=DVB-T+usb+stick+antenna}

[2] European Standard (Telecommunications series, ETSI en_300744v1.4.1, Digital Video Broadcasting (DVB), Framing structure, channel coding and modulation for digital terrestrial television 


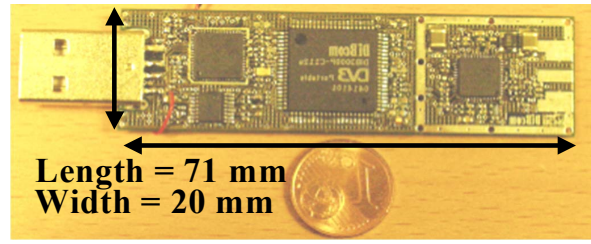

Figure 1: Photograph of the USB stick receiver

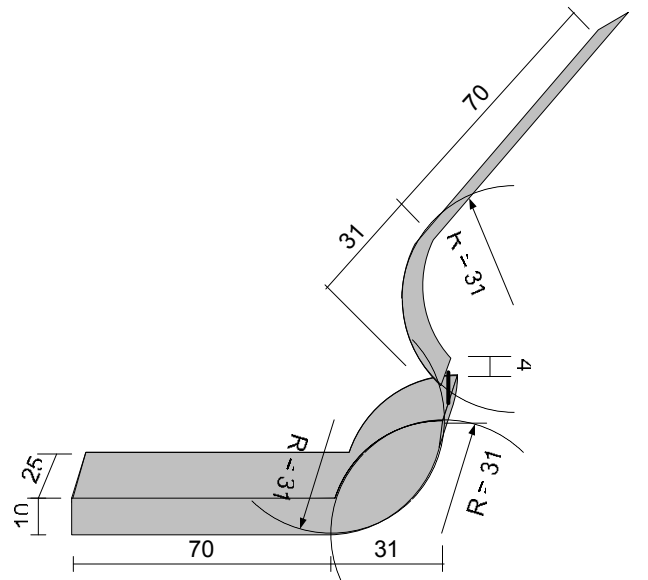

Figure 3 : Optimized antenna dimensions $(\mathrm{mm})$

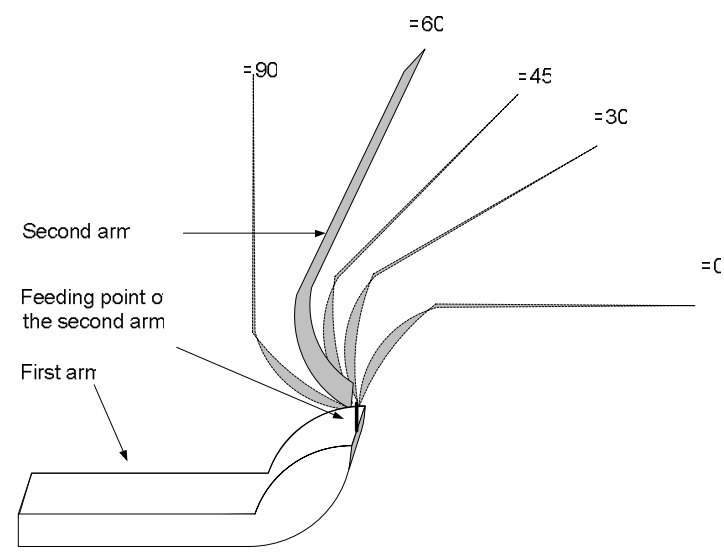

Figure $2: 3 \mathrm{D}$ perspective of the antenna with various positions of the second arm

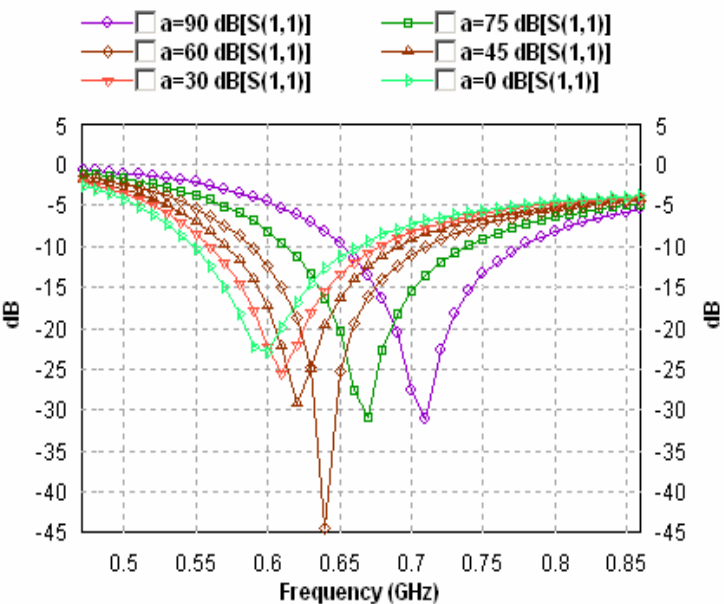

Figure 4 : Simulated S11 for various folding angles $\alpha$

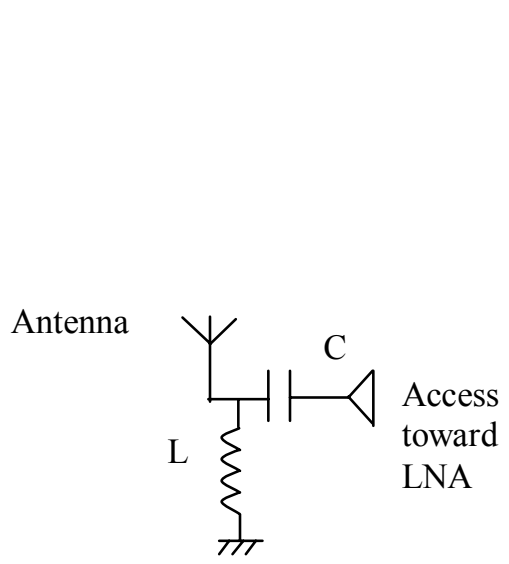

Figure 5 : Impedance matching network

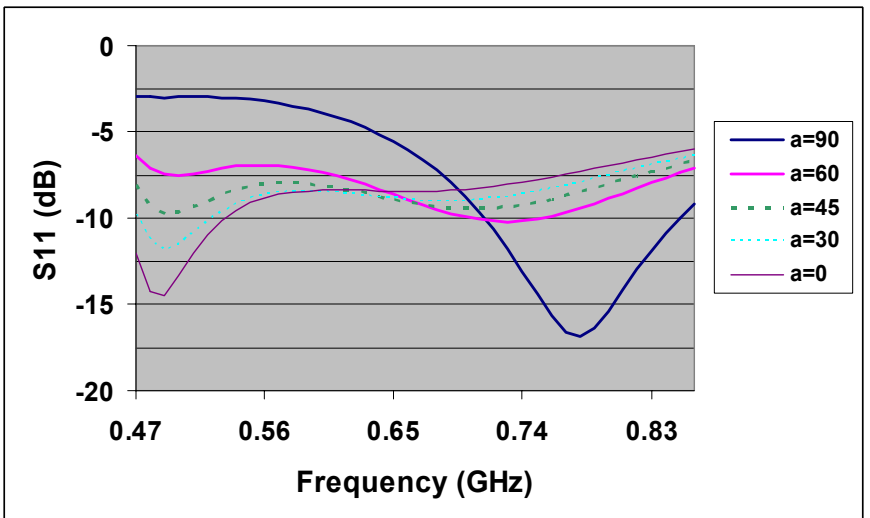

Figure 6 : Simulated return loss with impedance matching network for various folding angles $\alpha$ 
Figure 7 : Simulated antenna efficiency with folding angles $\alpha$ as parameter
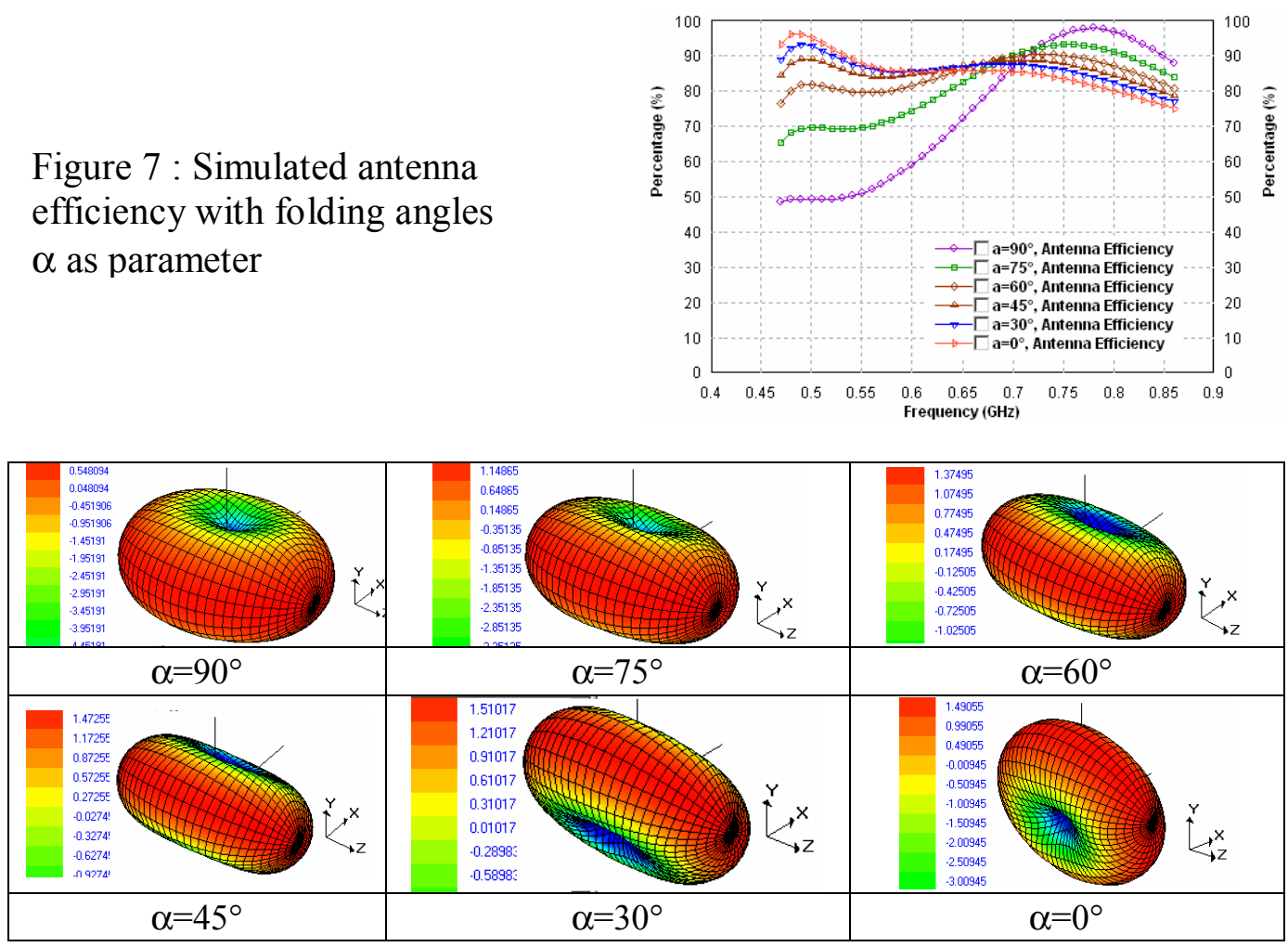

Figure 8: Simulated radiation patterns in gain for various folding angles $\alpha$ at $660 \mathrm{MHz}$

Figure 9 : Photograph of the working prototype

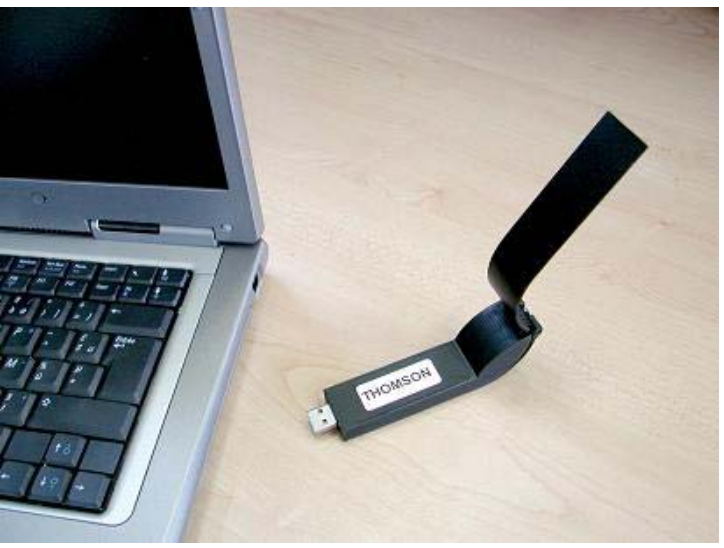

Figure 10 : Measured return loss for various folding angles $\alpha$

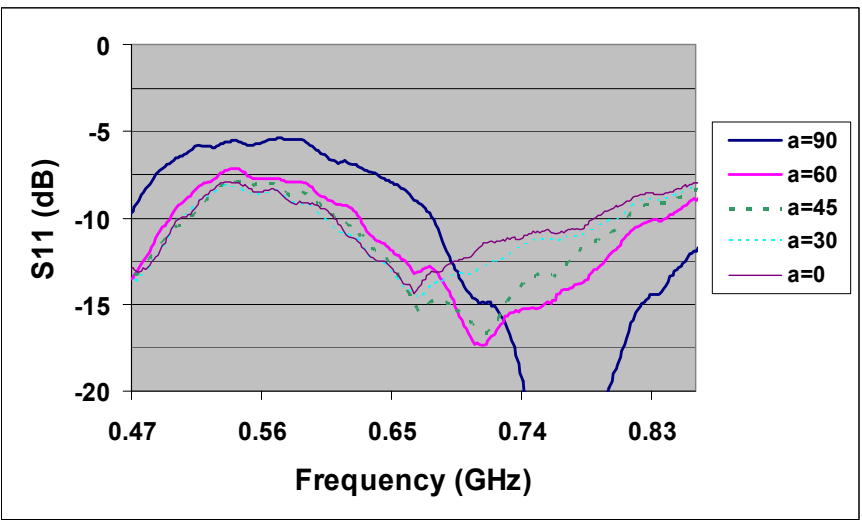

